\title{
Guardando ad Est: questioni di identità nelle coppie miste di italiani, polacche e rumene
}

EMILIANA BALDONI

UNIVERSITÀ DI FIRENZE

\section{Resumen}

Considerando el carácter cada vez más multicultural de nuestras sociedades, las parejas mixtas son una de los más relevantes indicadores de la integración de los inmigrantes en los países de destino, al tiempo que representan escenarios de encuentro y desencuentro de diferencias de toda índole (cultural, étnica, lingüística, religiosa, política, de clase, de ingresos, de nivel cultural, etc.). En este contexto se sitúa el presente artículo en el cual se estudia esta dinámica a partir de los discursos de parejas mixtas en Italia.

Palabras clave: parejas interétnicas, integración, multiculturalismo, migración

\begin{abstract}
Considering the increasingly multicultural character of our societies, the mixed unions are one of the more significant guides of integration of the immigrant community in the host countries. At the same time, we can observe different encounters and disencounters (cultural, ethnic, linguistics, religious, politics, of class, of income, of cultural level, etc.). In this context, the present paper analyzes these aspects across mixed couples in Italy.
\end{abstract}

Keywords: Inter-ethnic marriage, integration, migration, multiculturalism 


\section{Il fenomeno delle coppie miste in Italia}

Il fenomeno delle coppie miste, diffusosi in Italia in tempi relativamente recenti, costituisce uno dei mutamenti più rilevanti che stanno interessando i processi migratori nel nostro paese. Esso mostra "come cambiano i modi di fare famiglia in emigrazione e quali forme assume l'interazione e il confronto con l'altro" (Tognetti Bordogna, 1996 , 9). In una società che acquisisce sempre di più i caratteri del multiculturalismo, le unioni miste sono considerate tra gli indicatori più significativi di integrazione delle comunità immigrate nei paesi di destinazione; al contempo, esse rappresentano il terreno di incontro/scontro della diversità in tutte le sue declinazioni (culturale, etnica, linguistica, religiosa, politica, di classe, di reddito, di livello culturale, ecc.), una sorta di laboratorio di costruzione di nuovi significati, linguaggi, modalità di interazione e di decodificazione di vissuti lontani.

La definizione di coppie miste risulta tutt'altro che scontata e univoca. Il termine "misto" richiama immediatamente l'idea della differenza e della mescolanza. Tuttavia, è necessario chiarire a quale differenza fare riferimento. Nella letteratura anglosassone vengono utilizzati vari termini per descrivere il fenomeno dell'intermarriage: interracial marriage si riferisce a presunte differenze di "razza" (Tucker e Mitchell-Kernan, 1990; Pearlmann, 1997); interfaith marriage all'appartenenza a diversi credi religiosi; binational marriage alla provenienza da paesi diversi; interethnic marriage, infine, all'appartenenza a gruppi etnici diversi (Alba e Golden, 1986; Sanderfur, 1986), includendo così più caratteri contemporaneamente (provenienza geografica, religione, lingua, caratteri fenotipici, ecc.). I criteri che definiscono un'unione mista possono essere dunque di natura diversa ma la percezione di una differenza in termini di rilevanza è socialmente definita. Ciò significa che il grado di mixité di una coppia è determinato dal punto di vista di chi osserva il fenomeno ossia dal significato e dalla pertinenza che il contesto sociale attribuisce a certe differenze presenti nella coppia in un determinato momento (StreiffFenart, 1994; Gozzoli e Regalia, 2005). La mixité è quindi un concetto relativo, storicamente situato e influenzato dalle reazioni dell'ambiente circostante (Ambrosini, 2005); esso si configura come un costrutto sociale e culturale, variabile nel tempo e da cultura a cultura. 
La percezione della distanza tra contesti culturali diversi è inoltre mediata dallo status attribuito ai rispettivi gruppi d'origine. Per esemplificare, l'unione formata da un'italiana e un cittadino inglese o americano, in quanto appartenenti alla cultura occidentale dominante, è attualmente considerata - nel senso comune - "meno mista" di quella composta da un'italiana e un cittadino proveniente da un paese in via di sviluppo, ossia da una cultura ritenuta "minoritaria". Le differenze culturali vanno dunque analizzate anche all'interno delle dinamiche di potere che caratterizzano i rapporti tra i gruppi all'interno della società (Monacelli e Mancini, 2005).

A seguito dell'aumento dei flussi migratori verificatosi negli ultimi decenni, in Italia la presenza di cittadini stranieri, che secondo il Dossier Caritas-Migrantes 2007 ha raggiunto quota 3.690.000 (pari al $6,2 \%$ della popolazione totale), si è progressivamente diversificata fino a delineare un vasto e variegato panorama di provenienze geografiche, etniche e culturali. La tendenza di tale popolazione allo stanziamento definitivo trova riscontro in diversi indicatori, tra cui il significativo incremento del numero di unioni miste ${ }^{1}$. In base ai dati Istat (2005), nel 2001 le coppie miste nelle quali uno dei due partner è di cittadinanza italiana ammontano a 198.721, contro le 65.100 rilevate nel 1991; in tre casi su quattro $(76,1 \%)$ il partner straniero è una donna. Il fenomeno risulta maggiormente diffuso nel nord-ovest (32\%) e nell'Italia centrale $(25,8 \%)$ e, a livello regionale, in Lombardia e nel Lazio (rispettivamente 39.372 e 25.771 coppie). Analizzando la distribuzione per cittadinanza del partner straniero emerge che le prime cinque nazioni di provenienza nelle coppie "lui italiano-lei straniera" sono Germania (7,2\%), Francia (7\%), Romania (6,3\%), Polonia (5,6\%) e Brasile (4,9\%), mentre nelle coppie "lui straniero-lei italiana", oltre alla Germania $(7,9 \%)$ e alla Francia (7,6\%), prevalgono Marocco (6,6\%), Tunisia (6,4\%) e Regno Unito (6,3\%).

Se da un lato la predominanza in termini assoluti di unioni tra uomini italiani e cittadine tedesche e francesi è in parte il risultato di flussi di ritorno dell'emigrazione "maschile e celibe" italiana degli anni Sessanta diretta verso i paesi del Centro-Nord Europa, dall'altro

1 Le statistiche a cui fanno riferimento la maggior parte degli studi definiscono le coppie miste attraverso il criterio giuridico della nazionalità. 
risultano in netta crescita i matrimoni con donne provenienti dai paesi a forte pressione migratoria. Infatti, su 18.657 matrimoni misti tra uomini italiani e cittadine straniere celebrati nel 2005, nel 51\% dei casi la sposa è originaria dell'Europa centro-orientale (in particolare, Romania, Ucraina, Polonia e Russia) mentre nel 20,6\% proviene da un paese dell'America Latina ${ }^{2}$.

\section{Impianto metodologico della ricerca e profilo delle coppie italo-rumene e italo-polacche intervistate}

La presente ricerca, di tipo qualitativo, analizza il fenomeno delle unioni miste in Italia principalmente sotto una prospettiva identitaria prendendo in esame sedici coppie composte da un cittadino italiano e una cittadina di nazionalità rumena o polacca residenti nelle aree metropolitane di Roma e Firenze. Nell'ambito del processo di allargamento dell'Unione Europea, che apre scenari inediti e arricchisce di nuovi significati il senso di appartenenza all'Europa, si è deciso di focalizzare l'attenzione su immigrati provenienti da Polonia e Romania sia per la loro posizione di nuovi stati membri dell'Unione Europea, sia per la rilevante presenza numerica in Italia di cittadini di questi paesi. Inoltre, come già illustrato, in Italia le coppie miste formate da un italiano e una donna rumena o polacca compaiono al terzo e quarto posto dopo quelle italo-tedesche e italo-francesi. La necessità di accedere alla "definizione della situazione" del soggetto ha spinto ad utilizzare uno strumento di rilevazione caratterizzato da un basso livello di standardizzazione, quale l'intervista focalizzata. L'intervista, effettuata a ciascuno dei due coniugi separatamente (per un totale di trentadue interviste $)^{3}$, si articolava in sei aree finalizzate a rilevare:

1. il background socio-culturale dell'intervistato, ossia informazioni sul contesto familiare di origine, sul percorso formativo e lavorativo, sulla confessione religiosa, su altre eventuali esperienze di matrimonio o convivenza;

2 Nel 2005 sono stati registrati circa 23.500 matrimoni misti, pari al 9,6\% del totale (ISTAT, 2006).

3 Le coppie sono state individuate tramite un campionamento "a valanga" attivando reti personali e, in qualche caso, contattando associazioni o istituzioni rumene o polacche. Le interviste sono state sottoposte ad analisi qualitativa di tipo tematico. I nomi degli intervistati sono stati sostituiti con nomi di fantasia. 
2. per le coniugi straniere, l'intero percorso migratorio, dalla maturazione della scelta migratoria all'ingresso e insediamento in Italia;

3. la storia matrimoniale della coppia, con particolare attenzione alle circostanze di incontro e di celebrazione del matrimonio;

4. le scelte strategiche che riguardano la gestione del reddito familiare, il luogo di residenza, l'educazione dei figli, la sfera lavorativa;

5. gli aspetti salienti della vita quotidiana, con particolare riferimento alla gestione del tempo libero, alla lingua parlata in casa, alle reti sociali, all'osservanza di tradizioni del paese di origine, ai mezzi di comunicazione utilizzati, all'associazionismo;

6. l'identità (identità nazionale versus identità europea), l'orientamento politico e la sfera valoriale (in particolare, l'atteggiamento rispetto a tematiche chiavi quali l'aborto, il divorzio, l'omosessualità, le coppie di fatto, il fenomeno migratorio).

Le coppie intervistate si caratterizzano per una spiccata eterogeneità rispetto a gran parte delle variabili socio-demografiche considerate. L'età dei coniugi varia da un minimo di 28 anni a un massimo di 59 (per la moglie) e da un minimo di 30 anni a un massimo di 73 (per il marito). Complessivamente, le coppie italo-polacche risultano sposate in media da 15,5 anni; quelle italo-rumene da 19,6 anni. Più della metà delle coppie ha figli; inoltre, cinque intervistati (di cui quattro donne) hanno avuto precedenti esperienze di matrimonio o convivenza da cui sono nati altri figli ${ }^{4}$.

La scelta di concentrare l'attenzione solo sulle coppie legalmente sposate, escludendo le unioni di fatto, è riconducibile all'idea del matrimonio come possibile mezzo di regolarizzazione della posizione giuridica dei cittadini extracomunitari. In altre parole, si

4 In una coppia mista l'esperienza della genitorialità e, in particolare, l'educazione dei figli costituiscono il banco di prova per eccellenza delle dinamiche di negoziazione e di ibridazione culturale. Di questo fondamentale aspetto non è tuttavia possibile dar conto in questa sede. 
intendeva rilevare se l'eventuale mancanza del permesso di soggiorno avesse quantomeno accelerato la formalizzazione dell'unione, considerando la minaccia concreta di espulsione dal territorio italiano. Questo aspetto attraversa trasversalmente tutte le storie raccolte. Considerando solamente le coppie di recente formazione, in tre casi le coniugi straniere hanno esplicitamente definito il matrimonio in termini di "scelta forzata" dall'esigenza di ottenere i documenti di soggionno". Il senso di questo passo strategico è reso concisamente da Federica, rumena, 31 anni, sposata con Franco, 47 anni:

Ci siamo messi insieme il primo maggio e poi il 24 di luglio ci siamo sposati. Lui correva più di me. Più che altro ha voluto mettere le cose in chiaro... Forse ha avuto anche un po' di paura [ride] Non di paura, però ha detto: "Perché un domani ti posso perdere per una stupidaggine, se ti prende la polizia."

Analizzando la storia migratoria delle coniugi rumene e polacche emerge che in cinque casi il motivo di espatrio è stato il matrimonio, mentre nei restanti le intervistate sono emigrate principalmente per ragioni di lavoro, pressate talvolta da difficili condizioni economiche. Tra le testimonianze di emigrazione per "amore" è necessario segnalare quella di Lorena, che racconta di essere scappata dalla Romania con l'aiuto di un trafficante pagato dal marito. Il trasferimento in Italia per lavoro è avvenuto generalmente attraverso l'intermediazione di parenti o conoscenti che, attivando la rete etnica, hanno procurato un invito da parte di una famiglia italiana o un'offerta di lavoro nel paese di destinazione. Tutte le intervistate sono entrate in Italia con un visto turistico che, alla scadenza, ha generato una condizione di irregolarità. Il progetto migratorio originario era a breve termine; è stato poi l'incontro con il futuro coniuge a dirottare il corso dell'esistenza in direzione di una talvolta sofferta stabilizzazione, come racconta Lorena, rumena, 38 anni, sposata con Cristiano, 53 anni:

Prima abbiamo provato a sposarci regolarmente, ma $i$ tempi erano biblici... Abbiamo aspettato due anni e ancora niente... Allora lui ha trovato una persona

5 Tuttavia, al momento della rilevazione, le intervistate che avevano acquisito anche la cittadinanza italiana erano meno della metà. 
che... [ha un'esitazione] faceva passare la gente ai confini a pagamento... Io c'avevo una paura quando me l'ha detto! (...) Ho detto di sì, poi però mi sono pentita perché avevo paura. Se ti fermavano ti sparavano... Niente, sono scappata! Ho fatto trenta chilometri di notte a piedi, al confine rumeno con la ex Jugoslavia, poi al ponte diciamo in entrata in Jugoslavia mi aspettava lui con la macchina.

Il livello di istruzione delle coppie analizzate risulta prevalentemente medio/alto. Sette intervistate hanno conseguito la laurea nei paesi di origine mentre tra $i$ mariti il titolo di studio prevalente è il diploma di scuola media superiore. Solamente tre intervistati (due uomini e una donna) dichiarano di aver terminato gli studi con il completamento della scuola dell'obbligo. Tuttavia, l'impossibilità di convalidare il titolo di studio conseguito all'estero e le attuali condizioni del mercato del lavoro in Italia hanno determinato un inserimento lavorativo problematico, prevalentemente nel settore socio-assistenziale. La sensazione di disagio e frustrazione derivante dalla mancata realizzazione professionale emerge con forza in diverse testimonianze; in qualche caso, la rinuncia alle proprie ambizioni viene indicata con rammarico tra i "costi" più alti del matrimonio, insieme alla lontananza dagli affetti familiari. A fronte di ciò, va comunque rilevata la presenza di due intervistate impiegate l'una nell'istituto di cultura polacco, l'altra all'università come lettrice madrelingua, che al contrario esprimono piena soddisfazione rispetto alla loro condizione occupazionale. Un caso di frustrazione legato alle difficoltà di un adeguato inserimento occupazionale in Italia è quello di Marika, rumena, 57 anni, sposata con Marzio, 59 anni:

Io ho studiato liceo, poi l'università, ho fatto filologia, lingue il francese e poi ho insegnato, dopo ho conosciuto mio marito e sono andata via... purtroppo, diciamo, perché io stavo bene... insegnare ai bambini che mi volevano un mare di bene, questo rimpiangerò sempre [si commuove un $p 0^{\prime}$ ]. In Italia avrei dovuto rifare due anni di Università più la tesi (...) Allora un po' ho ricominciato a studiare poi non ce l'ho fatta. E qui in Italia non ho mai lavorato.

Un'analoga esperienza di mobilità professionale discendente viene testimoniata da Ramona, rumena, 50 anni, sposata con Fabio, italiano, 56 anni, che attualmente lavora come operatrice sanitaria specializzata presso un ospedale:

Ero ballerina all'Opera di Bucarest e insegnavo danza in una scuola popolare d'arte. Io prima di venire qui ho messo come condizione di poter lavorare (...) 
perciò una volta arrivata qui ho fatto l'esame da privatista per la terza media, per avere un titolo di studio che mi potesse dare almeno la possibilità di accedere ai concorsi pubblici.

La ricchezza del materiale empirico raccolto, che assume spesso le forme della narrazione biografica, si presta ad essere interpretato attraverso molteplici chiavi di lettura. In questa sede, in linea con gli obiettivi d'indagine, l'accento è stato posto principalmente su due tematiche cruciali, quali i percorsi di ridefinizione identitaria e i motivi della scelta matrimoniale.

\section{I processi di ridefinizione identitaria}

Le unioni miste rappresentano una zona di compatibilità delle differenze, uno spazio di contrattazione delle proprie specificità culturali attraverso un incessante lavoro di individuazione di equilibri provvisori rinegoziabili. Esse appaiono caratterizzate da una sorta di migrazione congiunta, ossia di attraversamento del proprio territorio di appartenenza al fine di stabilire un legame con l'altro (Gozzoli e Regalia, 2005). Tale percorso di migrazione simbolica comporta inevitabilmente la rielaborazione dei propri codici culturali e un progressivo percorso di ridefinizione identitaria. Risulta pertanto di estremo interesse esplorare le modalità con le quali l'identità etnica ascritta viene a riconfigurarsi nel contatto prolungato e costante con un'identità etnica altra e gli esiti di tali negoziazioni così come si manifestano nella vita quotidiana, nelle scelte di vita familiare, nell'educazione dei figli, nella socializzazione a simboli e pratiche etnicamente connotati (lingue, tradizioni, religione, relazioni sociali, comunità nazionali di riferimento nelle reti sociali primarie e secondarie).

A partire da tali considerazioni, la ricerca si è sviluppata attorno a due principali ipotesi guida. In primo luogo si suppone che la conciliazione delle identità etnico-nazionali avvenga tramite l'affermazione di fatto di una identità dominante e di una accessoria, eventualmente conservata in forma byphenated (ad esempio, la definizione della famiglia composta da coniugi italiani e polacchi come famiglia "italo-polacca"). La seconda ipotesi configura invece un processo di denazionalizzazione delle identità e di ancoraggio dei riferimenti valoriali e simbolici a contesti altri e nuovi, di segno universalistico e cosmopolita. In questa chiave, "l'Europa" si può 
affermare come area simbolica unificante nella misura in cui trascende le differenze di origine dei coniugi. In sostanza, per le donne rumene e polacche intervistate, l'esperienza di coppia con un partner italiano ha comportato più frequentemente l'assimilazione dei modelli culturali del paese ospitante e il confinamento della cultura d'origine in posizione accessoria o piuttosto l'adozione di strategie integrative? Una forte identificazione con l'Europa si verifica in maniera più marcata in assenza di attaccamento sia alla cultura di origine, sia a quella del paese di destinazione oppure nei casi di biculturalismo, ponendosi come identità aggiuntiva?

Numerosi studi sul fenomeno delle unioni miste fanno ricorso al concetto di acculturazione e ai modelli teorici di spiegazione dei processi di integrazione dei migranti nei contesti di insediamento. Le relazioni tra i coniugi possono infatti essere interpretate in termini di strategie di adattamento da parte del partner straniero alla cultura (maggioritaria) di quello autoctono. In questa sede, per esplorare il tema dell'identità etnica si è ritenuto opportuno ricorrere al modello bidimensionale di Berry (1980), che descrive quattro specifiche modalità di acculturazione, intesa come insieme di cambiamenti comportamentali e psicologici che si producono nell'individuo a seguito di contatti con persone appartenenti a differenti culture: l'assimilazione, il biculturalismo, la separazione e la marginalizzazione. Come mostrato in tabella, si ha assimilazione nel momento in cui un soggetto appartenente ad una minoranza abbandona la sua identità culturale e si identifica esclusivamente con la cultura del contesto di destinazione. Nel caso del biculturalismo, l'individuo mantiene le caratteristiche culturali del gruppo di provenienza (ad esempio, la lingua, gli usi e costumi, le tradizioni, gli stili di vita, le abitudini alimentari, ecc.) e, al contempo, acquisisce quelle della società maggioritaria. La separazione ha luogo quando il soggetto si identifica con proprio il gruppo etnico e si rifiuta di stabilire una relazione positiva con la società ricevente, mentre, infine, la marginalizzazione indica una condizione di perdita di identità senza che a ciò segua il tentativo di "accogliere" valori e credenze del gruppo ospitante.

L'applicazione del modello sopra esposto ha comportato la necessità di concentrare l'attenzione su una serie di fattori strategici quali l'autodefinizione della propria identità etnica, l'osservanza di usi e tradizioni del paese di origine, le relazioni sociali nel contesto di 
insediamento, i contatti con la comunità di connazionali, la rappresentazione del legame con il paese di origine e le scelte educative relative ai figli (in particolare, il bilinguismo).

Tabella 1.

\begin{tabular}{|l|c|c|}
\hline \multicolumn{1}{|c|}{ Tipi di acculturazione } & $\begin{array}{c}\text { Mantiene l'identità } \\
\text { culturale del paese di } \\
\text { origine? }\end{array}$ & $\begin{array}{c}\text { Ha relazioni positive } \\
\text { con la società } \\
\text { dominante? }\end{array}$ \\
\hline Assimilazione & NO & SI \\
\hline Biculturalismo/Integrazione & SI & SI \\
\hline Rifiuto/Separazione & SI & NO \\
\hline Marginalizzazione & NO & NO \\
\hline
\end{tabular}

Fonte: Berry $(1980,14)$.

Dall'analisi delle interviste è emerso innanzitutto che in poco più della metà dei casi la ridefinizione identitaria ha seguito prevalentemente strategie integrative. L'assunzione del biculturalismo può tuttavia essere intesa in una duplice accezione: in un senso forte come adozione di una "doppia identità culturale" o "doppia appartenenza", mantenendo una posizione di "equidistanza" da entrambe le culture di riferimento; in un senso più debole come piena identificazione con il paese di origine e acquisizione, al contempo, delle caratteristiche culturali del paese di destinazione.

Nel primo caso, meno frequente, le intervistate tendono a definire se stesse come "metà e metà" o "al confine tra due culture" che tuttavia non vengono vissute in modo contrapposto $\mathrm{e}$ inconciliabile. Tale condizione sottende l'idea che l'accettazione di un doppio patrimonio culturale costituisca comunque un arricchimento poiché amplia la gamma di codici interpretativi e comportamentali a cui attingere e favorisce l'elaborazione di sincretismi innovativi attraverso la rinegoziazione di tradizioni, valori e pratiche di ambedue le culture. Come illustrato di seguito da Angela e da Federica, tale operazione di bricolage culturale (Breger e Hill, 1998, 19-20) comporta la necessità di una presa di distanza da entrambi i contesti, per poterne poi riscoprire e valorizzare, nel confronto, aspetti positivi e negativi. 
Angela, polacca, 51 anni, sposata con Alberto, 54 anni:

Quando vado in Polonia mi sento italiana, quando sono qui mi sento polacca. [Della Polonia mi mancano] i rapporti fra le persone: molto vicini, da noi non c'è la solitudine. Qui invece sono tutti molto soli, in Polonia ci sono forti legami interpersonali in modo naturale che nessuno va dallo psicologo perché la tua psicologa è la tua vicina di casa.

Federica, rumena, 31 anni, sposata con Franco, 47 anni:

[Io mi sento] rumena di sangue, però italiana di... non lo so... Diciamo metà e metà (...) Vivendo qua, che c'bai il marito, una persona vicino a te che puoi parlare, puoi discutere le cose senza paura, senza nasconderti, senza che tu dici una cosa e la vede in un altro modo... Conta tanto questo.

Nell'accezione "debole" di integrazione, le intervistate esprimono un'identificazione esclusiva con le proprie radici ma al tempo stesso mostrano di aver acquisito in maniera non problematica anche i tratti culturali della società di accoglienza. In particolare, dichiarano di frequentare reti amicali italiane o miste (e non solamente comunità di connazionali), non osservano particolari tradizioni del paese di origine, prediligono i modelli comportamentali e gli stili di vita italiani, hanno trasmesso la loro lingua madre ai figli ma parlano con loro prevalentemente in italiano, ecc. In questo secondo caso, che riguarda esclusivamente intervistate di nazionalità polacca, l'identità dominante risulta essere quella d'origine mentre quella italiana sembra porsi piuttosto in posizione accessoria:

Iolanda, polacca, 41 anni, sposata con Patrizio, 45 anni

A questo punto io sono stata in Polonia lo stesso tempo che sono in Italia... mmm... io comunque mi sento sempre di appartenenza polacca, la mia origine, sono stati $i$ primi vent'anni (...) Le radici sono li. Poi mi sento italianizzata, sicuramente, perché vivo qui, faccio tutto qui.

Emilia, polacca, 34 anni, sposata con Davide, 37 anni

[Sento di appartenere a] tutti e due i paesi, non c'è uno più o meno, la Polonia per $i$ ricordi, per l'appartenenza comunque al paese, alla lingua. Devo dire che mi sento polacca, non mi sento italiana, però dall'altra parte [appartengo] all'Italia, perché mi offre una vita soddisfacente. Non sono italiana, ma sto bene in Italia e quindi mi interessa ciò che succede qua, vivo come qualsiasi italiano, le cose che accadono qua e vengono trasmesse in televisione diventano una discussione popolare, mi sento 
in qualche modo coinvolta. [Rispetto alle tradizioni culinarie] Fino all'anno scorso cercavo per forza di introdurre qualcosa di polacco, però veramente ho scoperto questa cosa, che non ha molto senso! Qui in Italia, non so se per il clima o per $i$ prodotti che uso, comunque non sono gli stessi che trovo in Polonia, l'effetto non è lo stesso e quindi mi sono detta che quando si va in Polonia, mi piace tanto, si mangia roba diversa.

Maria, polacca, 59 anni sposata con Silvano, 73 anni

Alcune volte quando sono un po' stanca dico a mio marito: "Ma cosa ho fatto io nella vita per avere due famiglie e due patrie" perché io sento questa responsabilità verso due patrie.

Vale la pena sottolineare che la messa in atto di strategie integrative trova riscontro in un atteggiamento di apertura e curiosità da parte dei coniugi italiani nei confronti della cultura rumena o polacca. Si tratta di intervistati che, oltre a conoscere la lingua, seguono con interesse la vita politica di quei paesi, apprezzano la cucina e intrattengono relazioni significative con i connazionali della consorte.

Il processo di assimilazione ha riguardato invece cinque intervistate, quasi tutte di origine rumena, le quali hanno dichiarato di sentirsi solo italiane e di non avere più alcun legame di appartenenza con il paese di origine. Ciò si riflette in una serie di aspetti tangibili tra $\mathrm{i}$ quali l'abbandono di ogni tipo di tradizione, l'assenza di contatti con la comunità di connazionali, il disinteresse verso la situazione economica e politica dei paesi di provenienza, fino alla rinuncia ad insegnare la propria lingua ai figli, nel timore che il bilinguismo, anziché costituire una ricchezza, possa provocare comportamenti discriminatori da parte dei coetanei italiani.

Lorena, rumena, 38 anni sposata con Cristiano, 53 anni

Adesso come adesso mi sento italiana. In tutto, nel modo di vivere... Quando sono arrivata qui facevo la tradurione, pensavo in rumeno e traducevo in italiano. Adesso il cervello usa direttamente l'italiano (...) Tradiqioni particolari... a dirti la sincera verità le ho perse, non me le ricordo più. Cucino solo italiano, anche perché ora la cucina rumena, che a loro non piace, mi sembra cosi pesante...

L'assunzione esclusiva dell'identità italiana presuppone una gerarchizzazione delle culture e si accompagna spesso ad una svalutazione del proprio patrimonio. Le posizioni più estreme lasciano ad intendere che la decisione di vivere in un altro paese comporti 
necessariamente un adeguamento totale alla mentalità e agli usi e costumi della maggioranza, ritenuti in ogni caso di "livello superiore". In tale processo un ruolo fondamentale è giocato dalle rappresentazioni negative che la società dominante proietta sui gruppi minoritari. Per due intervistate, infatti, l'identificazione con la cultura maggioritaria sembra il riflesso dell'interiorizzazione delle immagini negative più diffuse sui migranti rumeni, da cui entrambe mostrano di prendere nettamente le distanze. E' evidente che se le unioni miste contribuiscono all'integrazione dei vari gruppi etnici, ciò non indica necessariamente l'accettazione completa delle minoranze e il definitivo superamento di pregiudizi etnici.

Lucia, 29 anni, rumena, sposata con Gianni, 38 anni

Non è che posso venire qui a imporre la mia cultura, mi sembra una mancanza di rispetto. E poi lì sono ancora all'età della pietra! Per me lo straniero si deve adeguare alla maggioranza, non è che uno viene e impone la sua lingua e le sue tradizioni.

Marika, 57 anni, sposata con Marzio, 59 anni

In Romania non mi ritrovo più come mentalità, ho vissuto di più qui che in Romania e non mi ritrovo più con la mentalità dei rumeni assolutamente (...) sono abituata a ragionare piuttosto veloce, loro sono più lenti, più... vogliono rubare il tempo al tempo, nel senso che hanno sofferto cinquanta anni di comunismo ora però vogliono vivere come in Italia, allora rubano, fanno... non mi piacciono!

\section{Che cosa le manca di più del suo paese di origine?}

Niente!

\section{Niente?}

Zero. Perché la gente che viene oggi ci fa sentire male, la gente che è sul marciapiede a Milano, quelli che rubano bancomat e sono tutti rumeni e allora io in quei momenti mi vergogno di essere rumena.

Vi sono infine due casi emblematici, uno di separazione e l'altro di marginalizzazione. Nel primo, l'intervistata, pur ammettendo un certo attaccamento all'Italia, esprime un'identificazione esclusiva con la cultura di origine e mantiene un atteggiamento di forte contrapposizione (a tratti quasi di insofferenza) rispetto alla cultura italiana. Le sue parole richiamano direttamente la condizione di "estraneità" dello straniero già evidenziata da Simmel, in base alla quale 
lo straniero è riconosciuto come elemento della comunità ma ne è contemporaneamente di fronte e al di fuori (1908; tr. it. 1989, 580).

Daniela, polacca, 49 anni, sposata con Dario, 50 anni

Lo straniero se si adegua è visto un po' come un traditore della propria cultura. Non può diventare perfettamente inserito perché qualcosa si porta sempre dietro... E questo lo rende un po' sospetto... Se non si vuole adeguare, se rimane attaccato alle proprie tradizioni questo atteggiamento viene percepito come ostile. Quindi, sia la prima sia la seconda scelta si rivela sempre un po' sospetta. Si dà sempre un giudizio allo straniero (...) Io mi sento solo polacca. Il problema non me lo pongo proprio... Io sono cresciuta 24 anni a Varsavia, tutta la mia formazione, i miei ricordi, il mio modo di vedere il mondo, di pensare, è polacco... Non potrei sentirmi italiana perché non lo sono! L'attaccamento al luogo è un altro discorso. Quando io torno dalla Polonia sono contenta, perché questa per me è la mia casa. (...) Però con il passare del tempo alcuni aspetti della vita italiana li accetto sempre con maggiore difficoltà, ad esempio il caos, la noncuranza, la mancanza di rispetto per gli altri, atteggiamenti asociali.

Nel secondo, l'intervistata dichiara il suo allontanamento da entrambi i gruppi di riferimento, ribadendo di non sentirsi più "né rumena, né italiana"; le difficoltà di integrazione sono però spostate all'esterno e attribuite principalmente ad atteggiamenti discriminatori da parte degli italiani.

Any, rumena, 47 anni sposata con Andrea, 55 anni

Ti dico la verità [sento di appartenere a] nessuno dei due paesi, perché ora non sono né rumena né italiana. Vivendo qui e avendo la famiglia qua tutta insieme non è che più di tanto mi interessa lo Romania, sai se c'erano i genitori in vita era tutto un altro discorso ma come $i$ genitori non ci sono più... non me ne frega nulla, non c'è niente che mi attira! Ma qui quando sei straniero resti sempre uno straniero, puoi avere anche trenta o cinquanta anni di Italia, ma sei sempre uno straniero per gli italiani!

Per quanto concerne la seconda ipotesi di ricerca, si può innanzitutto osservare che, ad eccezione di due casi, le donne rumene e polacche intervistate esprimono un forte sentimento di appartenenza all'Europa, mentre gli italiani che dichiarano di "non sentirsi affatto europei" costituiscono quasi la metà del campione. Le motivazioni di tale disaffezione restano tuttavia piuttosto indeterminate. Per questi intervistati, l'Europa sembra configurarsi come un'entità astratta e 
fittizia, politicamente poco incisiva, costruita per tenere insieme contesti storico-sociali, culture e interessi in realtà del tutto lontani e inconciliabili.

Giorgio, 65 anni, sposato con Ilenia, rumena, 58 anni

Io mi sentivo europeo, anzi ero anche molto entusiasta, ma attualmente no, perché è una grande delusione l'Europa, non si occupa delle cose serie, non ba fatto niente dal punto di vista politico-militare, è solo un'unione monetaria e basta, serve a pagare gli euro faraoni.

Dario, 50 anni, sposato con Daniela, polacca, 49 anni

L'Europa non esiste, ho questa convinzione. Penso che sia un'identità falsa, una struttura costruita per altre ragioni, benché esista da secoli e sia riconosciuta come tale... Ma gli stati d'Europa non saranno mai uniti, perché quello che vuole un norvegese non coinciderà mai quello che vuole un portoghese, un greco, un turco.

La rivendicazione di un patrimonio comune fatto di valori, cultura, memoria storica, mentalità e stili di vita, caratterizza invece coloro che manifestano un forte senso di "europeismo". L'appartenenza all'Europa si manifesta anche per contrapposizione rispetto ad altre realtà politicamente ed economicamente dominanti (come ad esempio gli Stati Uniti e ciò che resta del blocco sovietico) e, in maniera ancora più marcata, rispetto a popoli e contesti percepiti come culturalmente lontani (in particolare, l'Africa). L'idea emergente (e auspicata!) dell'Europa è quella di un territorio unito privo di confini interni i cui cittadini possono finalmente esercitare il diritto di libera circolazione senza restrizioni.

Emilia, polacca, 34 anni, sposata con Davide, 37 anni

Io voglio sottolineare che in Europa la Polonia c'è stata sempre (...) Io mi sento europea perché vivo in Europa. Appartenere al continente europeo fisicamente e come cultura, perché penso che come cultura noi europei abbiamo tante cose in comune, stile di vita, modo di pensare, siamo diversi, dagli africani.

Marta, rumena, 40 anni sposata con Giuseppe 51 anni

$V$ edo tutti insieme, senza discriminazioni... Penso per le donne, per gli uomini, per la scuola, per il lavoro, anche per le religioni... Un'Europa unita in tutto... un pugno unito, senza frontiere, come in America, che uno può andare e venire come gli pare, può trovare lavoro ad esempio in Francia, senza problemi, senza permessi di soggiorno. 
Per concludere e tornare all'ipotesi di partenza, è importante precisare che l'identità europea si pone come "aggiuntiva" sia nei casi di donne "assimilate", sia in quelli di donne "integrate". Il fatto che quasi tutte le intervistate esprimano un'opinione positiva sull'Europa e sui processi di allargamento non sorprende, considerando gli indubbi vantaggi quantomeno in termini di mobilità. Le posizioni "più tenui" dei mariti fanno invece pensare che il matrimonio misto con cittadine dell'Est non sempre favorisca lo sviluppo di un pronunciato senso di appartenenza all'Europa. Si tratta comunque, è bene precisarlo, di osservazioni che riguardano il campione analizzato e che non hanno alcuna pretesa di generalizzazione.

\section{I motivi della scelta}

Le motivazioni alla base della scelta esogama costituiscono uno degli aspetti più studiati nella letteratura sulle coppie miste (Kalmijn, 1998, 396). Tale filone di ricerca, sviluppatosi a partire dagli anni ottanta del Novecento, si è andato evolvendo in linea con l'aumento dei flussi migratori e la stabilizzazione dei cittadini stranieri nei diversi contesti di destinazione. In generale, il processo di scelta di un partner straniero può essere considerato un fenomeno multidimensionale influenzato da diversi tipi di fattori interrelati (demografici, socioculturali, psicosociali, geografici) (cfr. Rodríguez, 2004). Tra le prospettive teoriche più recenti, che pongono l'accento ora sull'uno ora sull'altro ordine di cause, assumono particolare rilievo ai fini del presente lavoro la teoria strutturale e l'approccio motivazionale (cfr. Fenaroli e Panari, 2006, 45-9). Sulla base del primo approccio, di livello macrosociale, la formazione di matrimoni interculturali viene spiegata in funzione della composizione demografica di uno specifico paese ${ }^{6}$ e del forte incremento di opportunità di incontro tra persone provenienti da contesti culturali diversi, incremento legato alla mobilità sociale e geografica che caratterizza la società contemporanea (Gozzoli e Regalia, 2005, 160; cfr. anche Blau e Schwartz, 1984). Tale meccanismo è tuttavia legato ad una complessa trama di elementi (tra cui le relazioni politiche tra $\mathrm{i}$ due stati di appartenenza, il grado di integrazione delle

6 In riferimento al contesto statunitense, Lee e Edmonston (2005) osservano infatti che i matrimoni misti hanno maggiori probabilità di diffusione nelle aree più popolose $\mathrm{e}$ etnicamente eterogenee. 
comunità straniere nel paese di insediamento, il tempo di permanenza dei migranti, il livello di apertura o di pregiudizio dei diversi contesti, i significati attribuiti al matrimonio misto nelle diverse culture: cfr. Guyaux et al., 1992) i quali, in una sorta di circolo ricorsivo, contribuiscono alla creazione di un clima generale di maggiore accettazione e "normalizzazione" delle unioni non tradizionali (Fenaroli e Panari, 2006).

In una prospettiva di complementarietà, l'approccio motivazionale sposta invece l'attenzione sulle scelte individuali effettuate dalle singole persone, presupponendo che unioni miste e unioni omogame si formino in base a motivazioni sostanzialmente identiche. Se, come sottolinea Tognetti Bordogna (1996), in alcuni casi è evidenziabile una ragione prevalente, in altri è piuttosto l'interazione tra motivazioni diverse, relative sia al partner autoctono sia a quello straniero, a condurre all'unione interculturale.

Nell'ambito dell'approccio motivazionale vengono identificati due scenari principali: la differenza culturale e la somiglianza percepita (cfr. James e Tucker, 2003; Fu, 2001; Kalmijn, 1998). In estrema sintesi, nel primo caso a fondamento del matrimonio misto vi sarebbe proprio l'attrazione verso la diversità di cui l'altro partner è portatore, spesso in un'ottica "compensatoria" rispetto ad alcune caratteristiche specifiche (in particolare, la posizione sociale e il livello di istruzione). Già Merton (1941) e Davis (1941) prospettavano l'ipotesi di uno scambio di status all'interno del matrimonio misto nel momento in cui una persona appartenente ad una minoranza compensava l'acquisizione di uno status sociale più elevato dovuta al coniuge con altri requisiti (ad esempio, l'istruzione, un'attrattiva fisica, ecc.). Nell'approccio della somiglianza percepita, al contrario, la scelta del partner sarebbe motivata principalmente dalla ricerca di tratti comuni. Il matrimonio misto endogamico troverebbe dunque fondamento su caratteristiche di prossimità culturale, fermo restando un buon livello di accettazione reciproca tra rispettive culture di appartenenza. Un ulteriore approccio, denominato della scelta anticonvenzionale, spiegherebbe infine l'unione mista in termini di atto di ribellione alle regole e convenzioni relative al proprio gruppo di appartenenza. L'apertura verso il diverso assumerebbe dunque il significato forte di gesto di trasgressione e (spesso) rottura delle proprie tradizioni di origine (cfr. Fenaroli e Panari, 2006). 
Il riferimento all'approccio strutturale sopra delineato consente innanzitutto di ripartire le coppie intervistate in due gruppi. Il primo comprende unioni miste che si sono formate in tempi recenti, in concomitanza con il progressivo aumento dei flussi migratori dall'Europa dell'Est a partire dagli anni novanta. E' evidente che la forte presenza di migranti polacche e rumene e la loro concentrazione in determinati contesti territoriali (in particolare le aree metropolitane) hanno notevolmente accresciuto le possibilità di incontro, soprattutto considerando una percezione di distanza culturale relativamente ridotta tra le rispettive comunità di appartenenza, quantomeno nei confronti di persone provenienti da altri continenti o portatrici di marcatori etnici quali un diverso colore della pelle o una diversa religione. Il secondo gruppo riguarda invece quei matrimoni celebrati prima della caduta dei regimi comunisti e della conseguente apertura delle frontiere. Paradossalmente, quindi, è proprio la confutazione dell'approccio strutturale di partenza, in quanto si sono date unioni miste anche in assenza di opportunità concrete di incontro e di mobilità geografica, a fornire un primo criterio di classificazione delle coppie intervistate.

Nello specifico, nel campione intervistato sono presenti sette coppie che si formate prima del 1989. Nella maggior parte dei casi l'incontro è avvenuto in occasione di una vacanza da parte del coniuge italiano in Romania o in Polonia, mentre due intervistati italiani raccontano di aver conosciuto la propria consorte tramite rapporto epistolare $^{7}$. La storia di queste relazioni è stata segnata da momenti del tutto specifici: lunghi periodi di separazione intervallati da visite del partner italiano e dallo scambio di lettere, spesso sotto lo stretto controllo della polizia; complesse trafile burocratiche per l'ottenimento delle autorizzazioni e dei documenti necessari all'espatrio (concesso, nel caso della Romania, solo per motivi di matrimonio direttamente dal Consiglio di Stato $)^{8}$; ulteriori mesi di attesa e di separazione dopo la

7 Del resto, la pratica di "cercare moglie all'estero", in particolar modo in alcuni paesi dell'Est, era tutt'altro che rara per molti uomini italiani in condizione di "svantaggio" sul cosiddetto mercato matrimoniale. Al tempo stesso non può essere ignorata la diffusione di forme di turismo sessuale che, come affermato esplicitamente da un intervistato, erano alimentate (e contribuivano ad alimentare) stereotipi negativi e visioni distorte sulla presunta disponibilità delle donne dell'Est.

8 In un caso, come già evidenziato, l'espatrio è avvenuto in maniera illegale. 
formalizzazione dell'unione; infine, l'ingresso in Italia, rappresentato talvolta in termini di vero e proprio shock culturale?

Marika, rumena, 57 anni sposata con Marzio, 59 anni

Una mia amica corrispondeva con un ragazzo che faceva il militare con il mio marito e allora mio marito, che gli piace tanto i paesi stranieri in generale, ha detto: "Digli di mandare anche a me un indirizzo" e la mia amica ba mandato il mio indirizzo a mia insaputa, ho ricevuto la lettera poi ho risposto... Si sono scritte lettere, fotografie, cartoline per un anno e mezzo... e poi nel '70 d'estate è venuto. In un regime comunista non è che ci si sposa e si fa come si vuole, bisognava chiedere al Consiglio di Stato, praticamente al presidente Ceausescu (...) Ho messo $i$ documenti nel '72, ho ricevuto la risposta positiva nel '74, dopo sposata bo aspettato un altro anno perché lo stato ha pagato per me per farmi studiare e allora io dovevo ripagarlo con tre anni di insegnamento. Sicché finché non bo finito $i$ tre anni di insegnamento non mi hanno lasciato uscire dalla Romania.

Antonio, 55 anni, sposato con Antonella, rumena, 47 anni

Ho conosciuto mia moglie tramite degli amici... lei a questi amici ha fatto sapere che era una ragazza madre (...) Poi gli scrissi una lettera dicendo che io... la verità insomma, che sono intenzionato a portarla in Italia e a sposarla se lei contribuisce ad un certo tipo di aiuto perché io ho una piccola pensione, non potevo mantenerla, lei mi rispose che cercava l'uomo della sua vita ed è successo così e questa mi sembra l'anima gemella.

E' importante sottolineare ancora la peculiarità di questi rapporti "a distanza" sorti prima dell'apertura delle frontiere senza la possibilità concreta di una approfondita conoscenza reciproca. Le intervistate rumene tendono a sottolineare soprattutto la sofferenza con cui hanno vissuto l'ingerenza del regime sulla loro sfera personale. I mariti evidenziano piuttosto la "rischiosità" di questa scelta a "scatola chiusa", il timore di venire strumentalizzati o la valenza trasgressiva che il matrimonio misto ha assunto nei confronti delle regole del proprio gruppo di appartenenza, anche se non tutte le famiglie hanno reagito con iniziale ostilità e diffidenza.

9 In alcune testimonianze emerge chiaramente che il primo periodo di insediamento in Italia è stato accompagnato da un profondo senso di sradicamento, acuito dalla mancanza degli affetti familiari e dalla consapevolezza dell'impossibilità di visitare nell'immediato il paese di origine. 
Cristiano, 53 anni, sposato con Lorena, rumena, 38 anni

Un rapporto che ti conosci poco, è un punto interrogativo... E' un rischio! A quel tempo era una scatola chiusa anche per loro... Forse allinizio per lei non era questo grande amore, forse faceva il discorso: "Vado a stare meglio, questo è un bravo ragazzo". Io invece ero innamorato fin dall'inizio.

Dario, 50 anni, sposato con Daniela, polacca, 49 anni

Noi abbiamo fatto due matrimoni, prima a giugno con rito civile poi a settembre con rito religioso, e due feste, una più piccola e una più grande... Ma per la famiglia di mia madre era inconcepibile venire [in Polonia], perché significava in un certo senso avallare la mia decisione (...). All'inizio ho avuto pesi e obiezioni ma il fatto che io fossi cosi preso, ma proprio tanto, e cosi convinto, insomma l'ho fatto e basta. Poi dallintroduzione è cominciata la fase di accettazione. Io cercavo di dimostrare: "Guardate che i polacchi hanno una cultura"... Poi devo dire che l'esempio del Papa, grande presenza, ci ha aiutato tanto. Poi lei che ha iniziato a lavorare al Vaticano... Insomma, c'è stata all'inizio questa avversione, però subito ridimensionata dal prestigio... "Lavora al Vaticano, è laureata, ma quante lingue che sa! Ha amici e conoscenti che hanno ricoperto cariche importanti all'ambasciata, ai ministeri"... Tante cose che fanno prestigio, per cui tutta la famiglia per intero si è dovuta ricredere.

In questo quadro si inserisce l'analisi delle motivazioni individuali. Partendo dal presupposto che tutte le coppie intervistate si presentano come unioni di tipo elettivo, nelle quali l'affettività occupa il posto fondamentale, si è tentato di mettere a fuoco i fattori motivazionali che caratterizzano un'unione in un senso anziché in un altro. L'interrogativo di fondo che ha guidato l'analisi è il seguente: prendendo in considerazione tre variabili chiave (età, status socioeconomico di provenienza, livello di istruzione) nel campione intervistato il matrimonio misto è stato scelto più in funzione della somiglianza tra i partner o della diversità? L'ipotesi dell'esistenza di un meccanismo "compensatorio" reciproco rispetto ad alcune qualità rilevanti trova riscontro nelle testimonianze raccolte?

Innanzitutto, la differenza media di età tra i coniugi intervistati (6,8 anni) risulta sensibilmente più elevata rispetto a quella relativa alle coppie formate da cittadini italiani (pari a 3,6 anni, secondo i dati dell'ultimo censimento) (Istat, 2005). Emerge tuttavia una marcata distinzione in base alla nazionalità. Difatti, nelle coppie italo-rumene lo scarto è quasi doppio rispetto a quello relativo alle coppie italo- 
polacche (rispettivamente 9 contro 4,6 anni) ${ }^{10}$. Senza sovrastimarne l'importanza, offuscando altre caratteristiche personali determinanti, l'età anagrafica può essere dunque considerata tra i possibili fattori di compensazione se si assume da un lato che un'età più avanzata renda più difficoltosa la ricerca di un partner e dall'altro che la giovinezza costituisca una risorsa spendibile sul mercato matrimoniale in cambio di altre caratteristiche percepite come "meno prestigiose" (Bertolani, 2002, 27).

Federica, rumena, 31 anni, sposata con Franco, 47 anni

Beh, la differenza di età si vede ma a me non me ne frega niente, io lo amo così com'è. Il carattere, quello che c'ba dentro, vuol dire molto di più dell'aspetto fisico. Le prime parole sono state... pure questo mi ha colpito... ha detto: "Se cerchi la bellezza non la trovi in me". Queste sono state le prime parole di Franco.

Il confronto tra lo status socio-economico delle famiglie di provenienza di entrambi $\mathrm{i}$ partner (in termini titolo di studio e professione dei genitori, nonché di condizioni finanziarie complessive, anche alla luce della particolare situazione economica precedente e successiva al crollo delle economie pianificate nei due paesi dell'Est Europa) consente ulteriori considerazioni sulle possibili motivazioni alla base della scelta matrimoniale. In tredici coppie su sedici, infatti, i coniugi risultano appartenere a famiglie collocate su posizioni sociali diverse. Vale la pena sottolineare che tale disparità, trasversale alle due nazionalità considerate, volge nettamente a favore delle intervistate, le quali, nella maggioranza dei casi, provengono da contesti familiari caratterizzati da alti livelli di studio e di professionalità (genitori insegnanti, ingegneri, professori universitari, giuristi, psicologi, ecc.). E' altresì interessante osservare che i casi inversi (in cui lo status socioeconomico delle famiglie di origine delle partner straniere è più basso di quello delle famiglie dei mariti italiani) riguardano esclusivamente intervistate emigrate dal proprio paese alla ricerca di migliori condizioni di vita e di lavoro; in tali circostanze (tenuto conto anche della professione del partner italiano) il matrimonio misto ha effettivamente rappresentato un'opportunità di mobilità economica e sociale.

Emilia, polacca, 34 anni, sposata con Davide, 37 anni

10 In base ai dati Istat (2005), a livello nazionale la differenza di età nelle coppie formate da un italiano e una cittadina rumena o polacca è rispettivamente pari a 10,8 e 7,9 anni. 
Una volta tornando dall'ufficio di collocamento [prendeva un sussidio per ragazze madri] ho incontrato una signora che conoscevo, cosi parlando, mi è scappato detto che avrei voluto andare fuori, per guadagnare qualcosina per mettere da parte (...) l'idea era quella di partire in qualche posto, all'estero per due o tre mesi d'estate (...) per avere più sicurezza perché con $i$ soldi che guadagnavo là mi bastava appena appena per il mese, la base per vivere. E questa persona mi ha detto: "Guarda peccato che non ci siamo incontrate prima perché c'è mia sorella che lavora in Italia e magari ti poteva aiutare". E io ho detto: "Se la senti chiediglielo". Cosi mi richiama dopo due settimane e mi dice: "Guarda se vuoi vai che c'è il lavoro". Ed io sono stata presa un po' alle strette perché non è che per me doveva essere una cosa cosi immediata. E quindi niente, ho parlato con la mia mamma e lei ha detto: "Va bene", lei doveva essere d'accordo perché doveva prendersi la responsabilità di stare con mio figlio.

Fabio, 56 anni, sposato con Ramona, rumena, 50 anni

Al contrario di molte rumene di quel periodo, lei non aveva nessuna voglia di andare via dal suo paese! Aveva un bel gruppo di amici, e $i$ suoi genitori stavano non male.

Infine, l'analisi mostra che le coppie omogame dal punto di vista del livello di istruzione sono sei (cinque delle quali italo-rumene); nelle restanti unioni le coniugi straniere risultano aver conseguito un titolo più elevato rispetto al marito mentre la situazione inversa si è verificata solamente in un caso ${ }^{11}$. Questo risultato concorda con quanto emerso in diversi studi, i quali evidenziano che i membri di gruppi etnici minoritari con alto livello di istruzione si sposano più frequentemente con autoctoni meno istruiti (Kalmijn, 1998, 413). Quanto illustrato consente di concludere che, rispetto alle variabili chiave considerate, i matrimoni misti analizzati si fondano più su fattori motivazionali di attrazione verso la diversità che di somiglianza. In linea con altri contributi ${ }^{12}$, status socio-economico, età e livello di istruzione sembrano configurarsi come fattori di mitigazione della mixité, innescando all'interno della coppia inconsapevoli processi di riequilibrio delle risorse disponibili. Senza alcun intento di generalizzazione, è possibile dunque affermare che l'origine etnica delle

11 Nell'interpretazione di tale informazione va comunque tenuto conto del fatto che titoli di studio conseguiti in sistemi scolastici diversi non sempre sono equipollenti.

12 Cfr. in particolare Bertolani, 2002; Streiff-Fenart, 1988, 1989. 
coniugi straniere, percepita in termini di "condizione di svantaggio", sia compensata da caratteristiche personali considerate "socialmente prestigiose", o che, specularmente, il deficit del partner italiano in termini di istruzione, status socio-economico ed età sia compensato dall'appartenenza al gruppo maggioritario. Quale che sia la prospettiva adottata, entrambi i coniugi ne ricaverebbero un'impressione di miglioramento della propria condizione e di ascesa sociale.

\section{Note conclusive}

Il crescente aumento delle unioni miste è destinato ad apportare cambiamenti profondi nella società italiana. Nelle ottimistiche previsioni dei sostenitori della versione "classica" del paradigma assimilazionista, i matrimoni misti rappresentavano uno dei requisiti fondamentali per la realizzazione - attraverso una sorta di processo di amalgama biologico - del melting pot (Park, 1950) e dell'assimilazione strutturale degli immigrati negli USA (Gordon, 1964). Al di là delle valenze etnocentriche e prescrittive, che hanno decretato il definitivo fallimento del paradigma, resta comunque l'idea che le coppie miste contribuiscano in modo determinante all'integrazione dei vari gruppi etnici, anche se ciò non indica necessariamente l'accettazione completa delle minoranze culturali e il definitivo superamento dei pregiudizi etnici. Le riflessioni teoriche e gli studi empirici sull'argomento sono tuttavia scarsi nella letteratura italiana e si auspica che la riconosciuta rilevanza del fenomeno dia nel breve periodo forte impulso alla ricerca sociologica.

Anche se gli intervistati tendono a ridimensionare la distanza culturale e ad attribuire i conflitti a differenze caratteriali piuttosto che di status, l'analisi dei motivi della scelta ha mostrato, in linea con diversi contributi internazionali, il prevalere di una asimmetria tra i coniugi rispetto ad alcune variabili strutturali. Nell'analisi dell'aspetto identitario, invece, pur nei limiti della schematizzazione proposta, la prospettiva soggettiva in termini di autodefinizione si combina con la rilevazione di scelte e pratiche concrete in grado di svelare la qualità dell'attaccamento alla cultura di origine o di "adozione". Ne deriva un quadro complesso e sfumato, tutt'altro che schiacciato sul polo della cultura maggioritaria, in cui spesso la combinazione di elementi culturali diversi crea soluzioni originali e innovative. 
Rimane tuttavia in sospeso un interrogativo fondamentale: $i$ matrimoni misti tra cittadini italiani e neocomunitari contribuiscono concretamente alla costruzione dell'identità europea? Senza volersi addentrare nel controverso dibattito sulla definizione di tale concetto e sui suoi ambiti applicativi, in questa sede si può osservare che sebbene l'esistenza di legami forti tra cittadini comunitari, in particolare di tipo sentimentale, costituisca una delle condizioni fondamentali per lo sviluppo del senso di appartenenza alla società europea, nei casi analizzati la risposta sembrerebbe spostarsi più sul versante negativo. Difatti, se è pur vero che nonostante le posizioni più critiche dei coniugi italiani le intervistate esprimono una forte identificazione con l'Europa nei suoi aspetti storici e culturali, nel complesso emerge una scarsa partecipazione alla vita politica europea, una bassa frequenza di viaggi nei diversi paesi dell'Unione e una pressoché nulla adesione ad iniziative associative di mobilitazione per la difesa di interessi sovranazionali. 


\section{Riferimenti bibliografici}

Alba R.D., Golden R.M. (1986), "Patterns of Ethnic Marriage in the United States", Social Forces, 65, 202-223.

Alotta S. (2000), "Matrimoni misti: la scelta di un partner straniero", Studi Emigrazione/Migration Studies, XXXVII, 137, 41-66.

Ambrosini M. (2005), Sociologia delle migrazioni, Il Mulino, Bologna.

Barbara A. (1993), Les couples mixtes, Bayard, Paris.

Berry J.W. (1980), Acculturation as varieties of adaptation, in Padilla A. (ed), Acculturation. Theory, models and some new findings, Westview Press, Colorado, 9-25.

Berry J.W. (1989), Acculturation et adaptation psychologique in Retschitzki M., Bossel-Lagos, Dasen P.R. (eds), La reserche interculturelle, L'Harmattan, Paris, 135-145.

Bertolani B. (2002), Coppie miste a Reggio Emilia, Unicopli, Milano.

Blau P. M. and Schwartz J. E. (1984), Crossing Social Circles, Academic Press, Orlando.

Breger R., Hill R. (eds) (1998), Cross-cultural Marriage. Identity and Choise, Berg, Oxford.

Caritas-Migrantes (2007), XVII Rapporto sullimmigrazione. Dossier statistico 2007, Anterem, Roma.

Colja T. (2001), Trasmissione culturale e identitaria nei matrimoni misti. Analisi di una realtà concreta, Grafica Goriziana, Gorica, Zbirka Diplomska naloge.

Cottrell A.B. (1990), "Cross-national marriages: a Review of the Literature", Journal of Comparative Family Studies, XXI, 2, 151-169.

Davis K. (1941), "Intermarriage in Caste Societies", American Anthropologist, 43, 376-395.

Fenaroli P., Panari C. (2006), Famiglie "miste" e identità culturali, Carocci, Roma.

Fu V.K. (2001), "Racial intermarriage pairings", Demography, 38 (2), 147-159. 
Goody J. (1983), The Development of the Family and Marriage in Europe, Cambridge University Press, Cambridge.

Gordon M. (1964), Assimilation in American Life, Oxford University Press, New York.

Gozzoli C., Regalia C. (2005), Migrazioni e famiglie. Percorsi, legami e interventi psicosociali, Il Mulino, Bologna.

Guyaux A. et al. (1992), Double mixtes: la rencontre de deux cultures dans le mariage, L'Harmattan, Paris.

ISTAT (2005), Gli stranieri in Italia: analisi dei dati censuari, $14^{\circ}$ Censimento generale della popolazione e delle abitazioni, Edizione provvisoria, ISTAT, Roma.

ISTAT (2006), Rilevazione dei matrimoni. Rapporto annuale 2006.

James A., Tucker M. (2003), "Racial Ambiguity and Relationship Formation in the United States: Theoretical and Practical Considerations", Journal of Social and Personal Relationship, 20, (2), 153-69.

Kalmijin M. (1998), "Intermarriage and Homogamy: Causes, Patterns and Trends", Annual Review of Sociology, 24, 395-421.

Lee S.M., Edmonston B. (2005), "New Marriage, New Families: US Racial and Hispanic Intermarriage", Population Bulletin, 60 (2), 1-36.

Lievens, J. (1997), Interethnic Marriage: Bringing in the Context through Multilevel Modelling. Brussels and Ghent, Interuniversity Papers in Demography, IPD 1997-6.

Merton R. (1941), "Intermarriage and the Social Structure: Fact and Theory", Psychiatry: A Journal of the Biology and the Pathology of Interpersonal Relations, 4, 361-374.

Monacelli N., Mancini T. (2005), Appartenenze culturali e dinamiche familiari, in Fruggeri L. (a cura di), Diverse normalità, Carocci, Roma.

Park R.E. (1950), Race and Culture, The Free Press, Glencoe.

Pearlmann J. (1997), "Multiracials, Intermarriage, Ethnicity", Society, 23, 431-453.

Ratto M.G., Peirone M.G. (2003), Indovina chi viene a cena: matrimoni misti a Torino, B.Grande, Torino. 
Rodríguez D. (2004), Inmigración y mestizaje hoy. Formación de matrimonios mixtos y familias transnacionales de población africana en Cataluña, Publicaciones d'Antropologia Cultural, Universitat Autónoma de Barcelona, Barcelona.

Sanderfur G. (1986), "American Indian Intermarriage", Social Science Research, 15, 347-371.

Simmel G. (1908), Soziologie, Dunker \& Humblot, Berlin; tr. it. Cavalli A. (a cura di), Sociologia, Comunità, Milano, 1989.

Streiff-Fenart J (1988), "Les jeunes immigrées et le mariage mixte. Salut ou perdition?”, Peuples méditerranéens, 44-45, 137-154.

Streiff-Fenart J. (1989), Les couples franco-maghrébins en France, L'Harmattan, Paris.

Streiff-Fenart J. (1994), Problèmes de terminologie et ambiguitè de la notion, in Labat et al. (a cura di), Cultures ouvert, sociétés interculturelles: du contact à l'interaction, L'Harmattan, Paris, 226-232.

Therborn G. (1995), European Modernity and Beyond. The Trajectory of European Societies 1945-2000, Sage, London.

Tognetti Bordogna M. (1994), "Le famiglie Patchwork: matrimoni misti e ricongiungimenti familiari”, Marginalità e Società, 28, 1-18.

Tognetti Bordogna M. (a cura di) (1996), Legami familiari e immigrazione: i matrimoni misti, L'Harmattan Italia, Torino.

Tucker M., Mitchell-Kernan C. (1990), "New Trends in Black American Interracial Marriage: The Social Structural Context", Journal of Marriage and the Family, 52, 1, 209-218.

Varro G. (a cura di) (1995), Les couplet mixtes, Armand Colin, Paris. 